\title{
Autoimmunhepatitis und membranöse Glomerulonephritis unter Immuntherapie einer chronischen Hepatitis C
}

\author{
Maria Paparoupa, Ngoc Ahn Huy Ho, Frank Schuppert
}

Durch hochwirksame antivirale Medikamente ist die Hepatitis C von einer chronischen zu einer heilbaren Erkrankung geworden. Eine Infektion kann allerdings systemische und organspezifische Autoimmunerkrankungen auslösen, wie Vaskulitiden, hämolytische Anämie, Autoimmunhepatitis, Diabetes mellitus Typ 1 und Schilddrüsenerkrankungen [1]. In seltenen Fällen können sich diese auch erst während der Therapie manifestieren.

\section{Anamnese und initiale Diagnostik}

Unspezifische Beschwerden | Ein 63-jähriger Patient klagt über Müdigkeit und gibt an, seit Monaten an Gewicht zu verlieren. Gleichzeitig habe sich sein Allgemeinzustand verschlechtert. Um die Beschwerden abzuklären, wird er stationär aufgenommen. Außer einer milden arteriellen Hypertonie und einem insulinbehandelten Diabetes mellitus Typ 2 sind keine relevanten Vorerkrankungen bekannt. Der Patient gibt an, regelmäßig moderat Alkohol zu konsumieren.

Körperliche Untersuchung I Der Allgemeinzustand des übergewichtigen Patienten $(175 \mathrm{~cm}$, $92 \mathrm{~kg}$, Body-Mass-Index $30,0 \mathrm{~kg} / \mathrm{m}^{2}$ ) ist altersentsprechend. Die Körpertemperatur ist normal $\left(36,6^{\circ} \mathrm{C}\right)$, psychomotorisch gibt es ebenfalls keine Auffälligkeiten. Es ist kein Ikterus zu sehen, ebensowenig Leberstigmata der Haut. Das Abdomen ist weich und indolent sowie leicht adipös. Aszites oder eine Hepatosplenomegalie sind klinisch nicht nachweisbar. Die kardiopulmonale Auskulatation ist unauffällig. Weiterhin gibt es keine Hinweise auf eine Polyneuropathie oder rheumatologische Erkrankungen.

Routinelabor I Die Transaminasen und alkalische Phosphatase (AP) sind leicht erhöht:

- Glutamat-Oxalacetat-Transaminase (GOT): 80 U/1 (Referenzbereich: 5-34 U/1)

- Glutamat-Pyruvat-Transaminase (GPT): $131 \mathrm{U} / 1$ (Referenzbereich: < 55U/1)

- AP: 150U/1(Referenzbereich: 40-130U/1)
Alle weiteren Parameter sind im Normbereich, insbesondere auch das Carbohydrat-defiziente Transferrin (CDT; Desialotransferrin) mit 2,0\% (normal: bis 2,6\%)

Infektiologie | Antikörper gegen das Hepatitis-CVirus sind vorhanden, die Antigene und Antikörper für Hepatitis A und B sind negativ. Eine quantitative HCV-RNA-Reverse-Transkriptase-PCR ergibt eine Viruslast von $980000 \mathrm{IU} / \mathrm{ml}$, das HepatitisC-Virus (HCV) ist vom Genotyp 1b. Die Infektion dauert schätzungsweise länger als 6 Monate.

Endokrinologische und Autoimmundiagnostik | Die Schilddrüsenparameter

- Thyreoidea-stimulierendes Hormon (TSH),

- freies Trijodthyronin (fT3),

- freies Tetrajodthyronin(fT4),

- anti-TG,

- anti-TPO und

- TRAK

sind im Normbereich. Die ANAs sind mit einem Titer von 1:320 positiv. Negativ sind die Tests auf ANCA, AMA, LKM und SLA. SMA sind mit einem Titer von 1:40 nicht signifikant nachweisbar. Das Immunglobulin (Ig) G4 ist mit 1,1 g/l unauffällig, dasselbe gilt für IgG, IgA und IgM.

Ergänzende Untersuchungen | In der Sonografie des Abdomens zeigt sich ein pathologisches Leberparenchym im Sinne einer leichten Steatosis hepatis. Hinweise auf eine Leberzirrhose gibt es nicht. Die Nieren sind beidseits normal groß und unauffällig. Die sonografisch gesteuerte Bi-
Tab. 1 Die laborchemische Konstellation des nephrotischen Syndroms im Verlauf.

\begin{tabular}{|c|c|c|c|c|}
\hline & $\begin{array}{l}\text { Woche } 0 \\
\text { (Therapiebeginn) }\end{array}$ & Woche 21 & $\begin{array}{l}\text { Woche } 24 \\
\text { (Therapieende) }\end{array}$ & Woche 48 \\
\hline Kreatinin (mg/dl) & 1,1 & 1,1 & 1,2 & 1,1 \\
\hline Harnstoff (mg/dl) & 29 & 49 & 38 & 30 \\
\hline Gesamteiweiß Serum (g/dl) & 7,1 & 4,3 & 4,7 & 7,1 \\
\hline Gesamteiweiß Urin (mg/24 h) & negativ & 5,544 & negativ & negativ \\
\hline
\end{tabular}

\section{Glossar Antikörper AMA \\ anti-mitochondriale Antikörper \\ ANA}

anti-nukleäre Antikörper ANCA

anti-Neutrophile

cytoplasmatische

Antikörper

anti-TG

anti-Thyreoglobulin-

Antikörper

anti-TPO

anti-Thyreoperoxidase-

Antikörper

LKM

anti-Leber-Niere-Mikro-

somen-Antikörper

SLA

anti-lösliches-Leberantigen-Antikörper

SMA

anti-glatte-Muskulatur-

Antikörper

TRAK

Thyreotropin-RezeptorAntikörper 


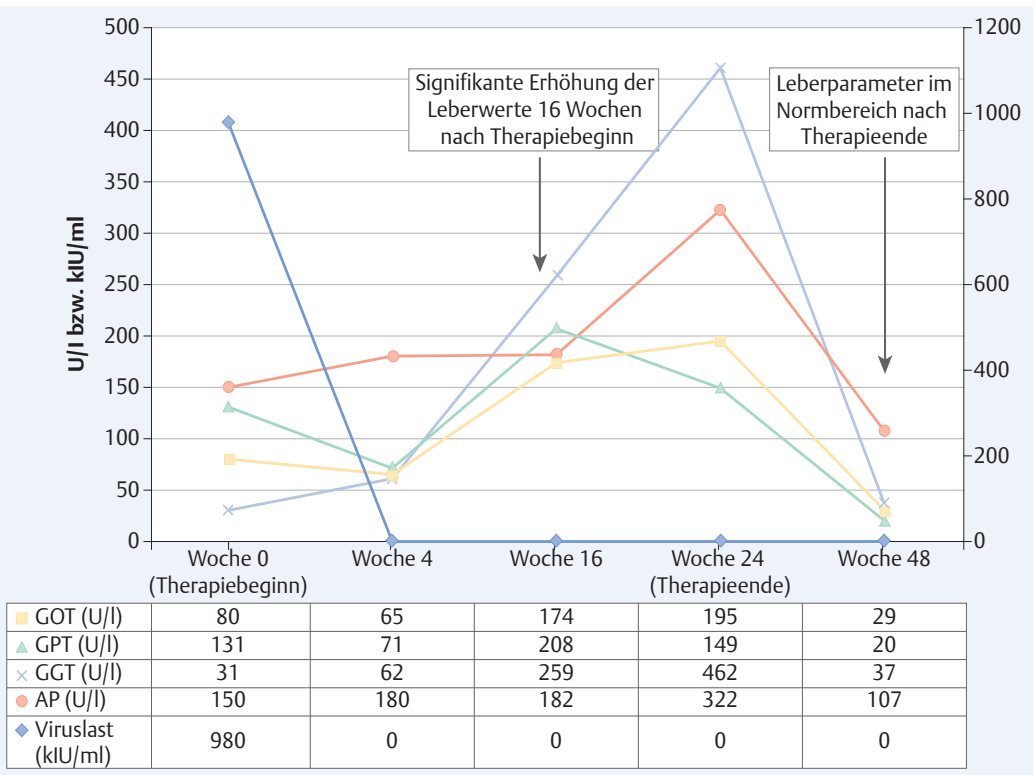

Abb. 1 Verlauf der Leberparameter und der Viruslast während der Therapie. GOT = GlutamatOxalacetat-Transaminase, GPT = Glutamat-PyruvatTransaminase, GGT = GammaGlutamyl-Transferase, $\mathrm{AP}=$ Alkalische Phosphatase

opsie der Leber ergibt eine milde, chronisch aktive Hepatitis C (Aktivitätsgrad II nach Desmet und Scheuer [2]) sowie eine mäßiggradige portale und sinusoidale Fibrose (Fibrose-Score 2 nach Desmet und Scheuer [2]). Der vorliegende Befund lässt kein morphologisches Korrelat einer Autoimmunhepatitis (AIH) erkennen. Weiterhin findet sich kein zirrhotischer Parenchym-Umbau und es gibt keinen Hinweis für Malignität.

\section{Therapie und Verlauf}

Tab. 2 Berechneter AutoimmunhepatitisGesamt-Score des Patienten 16 Wochen nach Therapiebeginn.

Geschlecht

Alkalische Phosphatase (U/I)

Glutamat-Oxalacetat- und

Glutamat-Pyruvat-Transaminasen (U/I)

Immunglobuline G über Normal

Autoantikörper ANA 1:320

Initiale Therapie | Bei nachweisbaren ANAs im Serum besteht formal differenzialdiagnostisch die Möglichkeit einer subklinischen, bisher inappa-

AMA nachweisbar

andere Autoimmunhepatitis-assoziierte Antikörper (pANCA, anti-SLA etc.)

Virushepatitis

Hepatotoxische Medikamente

Alkohol

HLA-DR3 / DR4 nachweisbar

andere Autoimmunerkrankungen

Therapieansprechen

Leberhistologie
Männlich

182

208

$<1,0 \times$

$1:>80$

nein

nein

ja

$<25 \mathrm{~g} / \mathrm{Tag}$

$-$

ja

biliäre Veränderungen komplett
Gesamt-Score $=1$ (Autoimmunhepatitis unwahrscheinlich) renten AIH. Dafür gibt es kein histologisches Korrelat und die restlichen leberrelevanten Autoantikörper sowie die Immunglobuline IgG, IgA und IgM sind normwertig. Initial erfolgt dennoch ein Therapieversuch mit nicht-pegyliertem Interferon-alfa-2a (IFN-alfa-2a, Roferon- ${ }^{\circledR}$ ). Nach 7 Tagen Behandlung sind die Leberparameter unverändert normal. Da im Verlauf einer Woche keine klinisch relevante AIH aufgetreten ist, wird die Therapie auf pegyliertes IFN-alfa-2a $(180 \mu \mathrm{g} /$ Woche) und Ribavirin (RBV; 1200 mg/ Tag) umgestellt.

Behandlung zunächst erfolgreich I Nach 4 Wochen ist die HCV-RNA unter der Nachweisgrenze. Die Leberparameter werden nach 8 und 12 Wochen Therapie kontrolliert und haben sich nicht signifikant verändert, sodass die Behandlung unverändert fortgesetzt wird.

Leberwerte steigen | 16 Wochen nach Therapiebeginn fallen bei der Routinekontrolle deutlich erhöhte Leberwerte auf ( Abb. 1). Bei positiven ANAs (Titer unverändert bei 1:320) sowie erhöhten IgGs (1640 mg/1; Referenzbereich: 700$1600 \mathrm{mg} / \mathrm{l}$ ) besteht nun die laborchemische Konstellation einer manifesten $\mathrm{AIH}$. Eine erneute sonografisch gesteuerte Biopsie der Leber kann eine akute oder chronische toxische hepatozelluläre Schädigung sowie das morphologische Korrelat einer AIH ausschließen. Die Therapie wird nach Rücksprache mit dem Patienten unter engmaschiger Kontrolle der Leberwerte fortgesetzt.

Weitere Verschlechterung | 21 Wochen nach Therapiebeginn stellt sich der Patient mit massiven Ödemen und Aszites notfallmäßig vor. Laborchemisch ist das Gesamteiweiß im Serum mit $4,3 \mathrm{~g} / \mathrm{dl}$ stark erniedrigt (Referenzbereich: 6,6$8,3 \mathrm{~g} / \mathrm{dl}$ ). Innerhalb von 24 Stunden scheidet er $5544 \mathrm{mg}$ Eiweiß im Urin aus. Aus den Befunden ergibt sich die Diagnose eines nephrotischen Syndroms ( Tab. 1). Es erfolgt eine sonografisch gesteuerte Biopsie der Niere. Bei der histologischen Untersuchung zeigt sich eine membranöse Glomerulonephritis (überwiegend Stadium I, segmental auch Stadium II nach Ehrenreich [3]) ( Abb. 2 und Abb. 3 ).

Therapie I Da die Nierenfunktion nur moderat eingeschränkt ist (glomeruläre Filtrationsrate: $65 \mathrm{ml} / \mathrm{h}$; Referenzbereich: $>85 \mathrm{ml} / \mathrm{min}$ ) und die Nierenretentionsparameter aktuell nicht angestiegen sind, verzichten wir auf eine immunsuppressive Therapie. Trotz der initialen Viruslast von $980000 \mathrm{IU} / \mathrm{ml}$ wird die antivirale Therapie nur für 24 Wochen durchgeführt und nicht, wie ursprünglich geplant, für 48 Wochen. Nachdem die Behandlung mit pegyliertem IFN-alfa-2a und RBV abgeschlossen ist, liegt die Viruslast unterhalb der Nachweisgrenze. Die Leber- und Nierenparameter haben sich vollständig normalisiert $(\triangleright$ Abb. 1 und $\triangleright$ Tab. 1 ). 


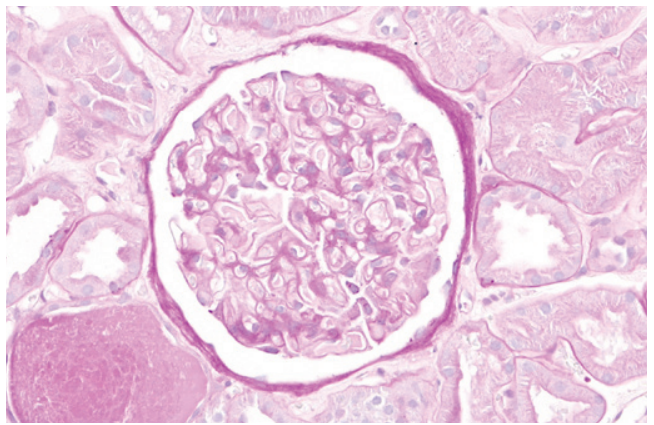

Abb. 2 Lichtmikroskopische Aufnahme eines Glomerulus mit starren und breiten Basalmembranen; Mesangium und Endothelien regelrecht (Periodic-acidSchiff-Reaktion-Färbung; 200×).

\section{Diskussion}

Phänomen bekannt | AlHs, die sich während oder nach einer antiviralen Therapie mit IFN entwickeln, sind ausführlich in der Literatur beschrieben [4]. Die Erkrankung kann auch mehrere Jahre nach dem Ende dieser Behandlung auftreten [5]. Manchen Autoren zufolge sollte ein positiver ANA-Titer vor Therapiebeginn die Entscheidung, eine IFN-Therapie einzuleiten, nicht beeinflussen, da die Effektivität der Behandlung nicht geringer ist [6]. Unser Fall zeigt, dass eine Therapie mit IFN das Risiko für Autoimmunphänomene erhöhen kann. Dies gilt insbesondere für prädisponierte Patienten, die z.B. positive Autoantikörpertiter aufweisen.

Unentdeckt durch geringe Ausprägung | In unseren Fall konnte eine retrospektiv präexistente, subklinische $\mathrm{AIH}$ nicht detektiert werden, obwohl zunächst testweise nicht-pegyliertes IFNalfa-2a verabreicht wurde. Erst die Therapie mit pegyliertem IFN über 16 Wochen induzierte bzw. aggravierte die $\mathrm{AIH}$, sodass diese laborchemisch apparent wurde. Allerdings reichte der berechnete AIH-Score des Patienten [7] nicht, um diese zu beweisen $(\checkmark$ Tab. 2), und ein histologisches Korrelat lag auch nicht vor. Dies ist wahrscheinlich bemessen an den nur leicht erhöhten IgG-Globulinen - auf die geringe Ausprägung der $\mathrm{AIH}$ zurückzuführen.

HCV-assoziierte Nierenschäden | Im Zusammenhang mit der HCV-Infektion können zudem verschiedene Pathologien der Niere auftreten. Der häufigste derartige histologische Befund ist die Typ-I-membranoproliferative Glomerulonephritis mit Typ-II-Misch-Kryoglobulinämie [8]

Medikamenten-induzierte Nierenschäden | Einige antivirale Medikamente, einschließlich der Kombination aus pegyliertem IFN-alfa-2a und RBV, können laut Literatur nephrotoxisch wirken [9]. Der genaue Mechanismus ist noch nicht vollständig geklärt. Sekundäre Formen der medikamentös induzierten Glomerulonephritis können

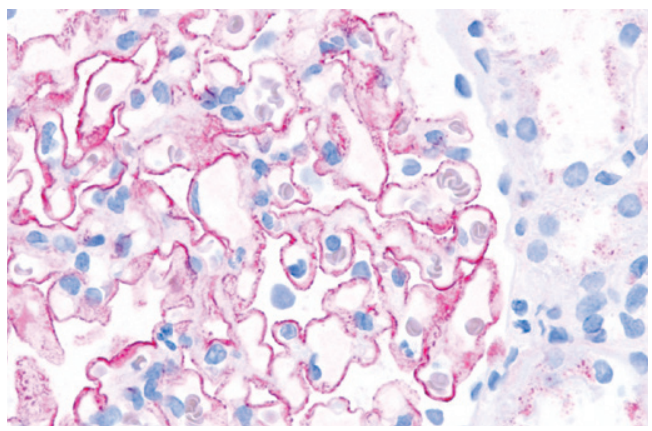

Abb. 3 Immunhistologie für Immunglobulin G mit granulärer Positivität entlang glomerulärer Basalmembranen (Alkaline-Phosphatase-anti-Alkaline PhosphataseFärbung; 400x).

zusätzlich das klinische Bild bei Patienten mit Nierenbeteiligung erschweren.

Auslöser wahrscheinlich IFN I In unserem Fall trat die Nephropathie erst nach 21 Wochen unter der Therapie mit pegyliertem IFN-alfa-2a und RBV auf. Nach dem Ende der Behandlung normalisierten sich die Werte rasch. Wir gehen daher von einer IFN-induzierten Glomerulonephritis aus. Bei bestehender HCV-Infektion wäre auch eine Hepatitis-assoziierte Glomerulonephritis differenzialdiagnostisch zu erwägen. Dies ist jedoch unwahrscheinlich, da sich die renale Schädigung unter virussuppressiver Therapie nicht verbesserte, sondern verschlimmerte. Zudem war bei dem Patienten zu diesem Zeitpunkt keine HCV-RNA mehr nachweisbar. Letzlich kann eine Kryoglobulinämie aber nicht sicher ausgeschlossen werden.

\section{Konsequenz für Klinik und Praxis}

- Mit Autoimmunreaktionen unter einer antiviralen Medikation mit Interferon (IFN) sollte man vor allem bei genetisch prädisponierten Patienten mit positiven Autoimmunmarkern rechnen.

- Die Autoimmunität kann so hochgradig und unbeherrschbar sein, dass die Therapie vorzeitig beendet werden muss.

- Der Patient sollte klinisch und laborchemisch überwacht werden, da einige Nebenwirkungen erst nach längerer Anwendung auftreten können.

- Da es heutzutage alternative, IFN-freie Therapieoptionen gibt, muss der Einsatz von IFN angesichts solcher nebenwirkungsreicher Therapieverläufe mit Autoimmunität kritisch gesehen werden.

Wir danken Herrn Professor Herman-Josef Gröne, DKFZ Heidelberg, für die Fotografien und die Beschriftungen der Nierenhistologien sowie für die kritische Durchsicht des Manuskripts. Ebenso danken wir Herrn PD Dr. Markus Cornberg, Medizinische Hochschule Hannover, für die kritische Durchsicht des Manuskripts.

Vollständiges Literaturverzeichnis unter http://dx.doi.org/10.1055/s-0041-106681

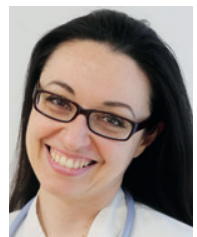

Maria Paparoupa, MD, MPH

ist Assistenzärztin an der Klinik für Gastroenterologie, Endokrinologie, Diabetologie und Allgemeine Innere Medizin, Zentrum für Viszeralmedizin und Onkologie, Klinikum Kassel. maria.paparoupa@yahoo. com

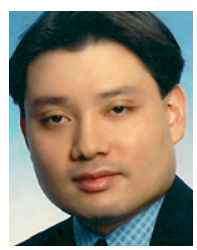

Dr. med. Ngoc Ahn Huy Ho ist Oberarzt an der Klinik für Gastroenterologie, Endokrinologie, Diabetologie und Allgemeine Innere Medizin, Zentrum für Viszeralmedizin und Onkologie, Klinikum Kassel.

ngoc.ho@klinikum-kassel.de

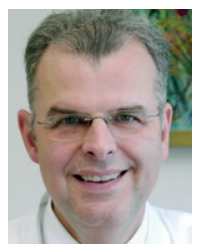

Prof. Dr.med.

Frank Schuppert

ist Direktor der Klinik für Gastroenterologie, Endokrinologie, Diabetologie und Allgemeine Innere Medizin, Zentrum für Viszeralmedizin und Onkologie, Klinikum Kassel.

frank.schuppert@klinikumkassel.de

\section{Interessenkonflikt}

Die Autoren geben an, dass kein Interessenkonflikt besteht.

DOI 10.1055/s-0041-106681

Dtsch Med Wochenschr 2016; 141: 709-711

(c) Georg Thieme Verlag KG . Stuttgart · New York . ISSN 0012-0472 


\section{Literatur}

1 Calvaruso V, Craxì A. Immunological alterations in hepatitis C virus infection. World J Gastroenterol 2013; 19: 8916-8923

2 Desmet V], Gerber M, Hoofnagle JH et al. Classification of chronic hepatitis: diagnosis, grading and staging. Hepatology 1994; 19: 1513-1520

3 Ehrenreich T, Churg J. Pathology of membranous nephropathy. Pathol Ann 1968; 3:145

4 Kogure T, Ueno Y, Fukushima K et al. Fulminant hepatic failure in a case of autoimmune hepatitis in hepatitis $C$ during peg-interferon-alpha $2 \mathrm{~b}$ plus RBV treatment. World ] Gastroenterol 2007; 13: 4394-4397

5 Efe C, Heurgué-Berlot A, Ozaslan E et al. Late autoimmune hepatitis after hepatitis $C$ therapy. Eur I Gastroenterol Hepatol 2013; 25: 1308-1311

6 Khairy M, El-Raziky M, El-Akel W et al. Serum autoantibodies positivity prevalence in patients with chronic HCV and impact on pegylated interferon and RBV treatment response. Liver Int 2013; 33: 1504-1509

7 Alvarez F, Berg PA, Bianchi FB et al. International Autoimmune Hepatitis Group Report: review of criteria for diagnosis of autoimmune hepatitis. ] Hepatol 1999; 31: 929-938

8 Ozkok A, Yildiz A. Hepatitis C virus associated glomerulopathies. World J Gastroenterol 2014 28; 20: 7544-7554

9 Murakami C, Melda Urekli $\mathrm{H}$ et al. Antiviral medications for the treatment of hepatitis $B$ and $C$ infection and their effects on kidney function. 